\title{
Strain path and work-hardening behavior of brass
}

\author{
N.A. Sakharova ${ }^{a}$, J.V. Fernandes ${ }^{a}$, M.F. Vieira ${ }^{b, *}$ \\ a CEMUC, Department of Mechanical Engineering, University of Coimbra, Rua Luís Reis Santos, Pinhal de Marrocos, 3030-788 Coimbra, Portugal \\ ${ }^{\mathrm{b}}$ CEMUC, Department of Metallurgical and Materials Engineering, Faculty of Engineering of the University of Porto, Rua Dr. Roberto Frias, $4200-465$ Porto, Portugal
}

\section{A R T I C L E I N F O}

\section{Article history:}

Received 13 May 2008

Received in revised form

20 November 2008

Accepted 4 January 2009

\section{Keywords:}

Deformation

Mechanical properties

Electron microscopy

Microstructure

\begin{abstract}
A B S T R A C T
Plastic straining in metal forming usually includes changes of strain path, which are frequently not taken into account in the analysis of forming processes. Moreover, strain path change can significantly affect the mechanical behavior and microstructural evolution of the material. For this reason, a combination of several simple loading test sequences is an effective way to investigate the dislocation microstructure of sheet metals under such forming conditions. Pure tension and rolling strain paths and rolling-tension strain path sequences were performed on brass sheets. A study of mechanical behavior and microstructural evolution during the simple and the complex strain paths was carried out, within a wide range of strain values. The appearance and development of deformation twinning was evident. It was shown that strain path change promotes the onset of premature twinning. The work-hardening behavior is discussed in terms of the twinning and dislocation microstructure evolution, as revealed by transmission electron microscopy.
\end{abstract}

(c) 2009 Elsevier B.V. All rights reserved.

\section{Introduction}

Fundamental aspects of the plastic deformation of metals have been established by extensive studies of both single crystals and polycrystals. Early studies of single crystals of materials with high stacking fault energy (SFE) established that, during deformation, dislocations tend to arrange themselves in cell walls, showing close correlations to active slip systems [1,2]. For single crystals, the shape of the dislocation cells is related to the number of active slip planes: at the beginning of deformation when one slip system is clearly more active than the others, one family of dislocation walls is observed; once two slip systems become active, two intersecting families of parallel dislocation walls, delimiting closed parallelogram cells, are generally observed in the crystals; and the equivalent activation of three or more slip system induces the formation of closed equiaxed cells.

During deformation of polycrystalline metals, internal stresses due to the accommodation process between adjacent grains act on the plastic behavior. Multiple slip is then imposed inside the grains, whatever their orientation. In materials with high SFE, closed dislocation cells (four-sided or equiaxed) are the predominant feature in grains of a polycrystalline sample, depending on the grain size and type of strain path [3-6]. Comparison between cell wall orientations and the Sachs model calculations of the slip systems may indicate the level of intragranular stresses and the homogeneity of the intragranular stress field.

\footnotetext{
* Corresponding author. Tel.: +351 225081424; fax: +351 225081447.

E-mail address: mvieira@fe.up.pt (M.F. Vieira).
}

The plastic behavior of metals after strain path change has been widely studied, mainly in mild steel [3], copper [5-8], aluminum [9], and brass [10]. Active slip systems are often observed to change partially or totally after reloading. The activation of new slip systems, typical of the reloading path, depends on the magnitude of the strain path change. This is usually defined by a scalar parameter $v$, initially proposed by Schmitt et al. [11]: $v=\left(\varepsilon_{\mathrm{p}}: \varepsilon\right) /\left(\left\|\varepsilon_{\mathrm{p}}\right\|\|\varepsilon\|\right)$, where $\varepsilon_{\mathrm{p}}$ and $\varepsilon$ are the plastic strain tensors corresponding respectively to the prestrain and the subsequent deformation. The value of $v$ is merely the cosine of the angle between the two vectors that represent successive strain tensors. The activation of new slip systems in the grains increases when $v$ evolves from 1 to 0 . In fact, for $v=1$, there is no change of strain path and the previously active slip systems are still active after reloading. Consequently, no significant changes in the mechanical behavior after path change are detected for $v=1$.

For $v=0$, the strain path change is drastic, i.e. most of the slip systems activated after reloading have not been activated during the first path. The mechanical behavior in the second strain path can be characterized by the ratio $\sigma_{\text {be }} / \sigma_{\mathrm{r}}$ (where $\sigma_{\text {be }}$ is the reloading yield stress and $\sigma_{\mathrm{r}}$ is the stress measured at the end of the first path) and by the relative value of the work-hardening rate, i.e. comparing, at a given stress, the work-hardening rate after reloading with the one for the simple tension.

Therefore, for $v=0$ latent hardening effects become significant and $\sigma_{\text {be }} / \sigma_{\mathrm{r}}$ can take values close to 1.12 (copper under tension after normal rolling [7], for example). Moreover, a relatively low work-hardening rate is observed in the early stage of reloading, mainly due to the dynamic recovery of the previous dislocation structures, which is stronger when active slip systems are modified. 
The dislocation structure becomes unstable when the deformation conditions change: the passage of groups of dislocations into dislocation tangles and cells tends to change their organization and promotes the annihilation of dislocations [3-5]. The previous dislocation structure is gradually replaced by another structure, typical of the new path. This structure has greater stability under the new loading conditions and, as a result, the mechanical behavior tends towards the one representative of the current path.

During plastic deformation, the occurrence of twinning, as an additional mechanism to dislocation slip, considerably changes some of the usual features of deformation. This takes place for materials with low-to-moderate stacking fault energy. Most materials do not twin until appreciable plastic deformation by dislocation slip has taken place [12-14]. The activation of deformation twins competes with dislocation slip and affects the mechanical response of the material. The initiation of the deformation twinning mechanism depends on the ratio between the critical resolved shear stresses (CRSS) for twinning and for slip [12,15,16].

The deformation behavior of FCC metals and alloys with lowto-moderate SFE under different straining conditions has mainly been studied in copper [17,18], stainless steel $[19,20]$ and brass [20-24]. Shock deformation produces deformation twins in copper [18]. Examples of such dynamic deformation microstructure in wide class of FCC materials subjected to high velocity and shock deformation have recently been investigated (for example [20]). For stainless steel $[19,20]$ and brass [20-24], deformation twins were observed for low deformation velocity under simple tension, plane strain compression and simple shear. Also, a few studies concerning sequential strain paths, particularly tensile-cyclic torsion of $70 / 30$ brass and stainless steel [21,22] have been performed. However, the effect of the type of strain path change on materials deformed by twinning has never been studied, to our knowledge.

The aim of this study is to investigate the mechanical behavior and microstructural evolution of $70 / 30$ brass sheet, deformed by simple tension and simple rolling and the complex strain paths of normal $(v=0)$ and parallel $(v=0.87)$ tensions after prestrain in rolling. The microstructural results, obtained by transmission electron microscopy (TEM) are correlated to the mechanical behavior.

\section{Experimental procedure}

The material used in this study is $70 / 30$ brass, which is typically representative of low SFE alloys $\left(\gamma_{\mathrm{sf}}=7 \mathrm{~mJ} / \mathrm{m}^{2}\right)$. The samples were cut from a cold-rolled and annealed sheet, $3 \mathrm{~mm}$ thick and with an average grain size of $20 \mu \mathrm{m}$, and then were annealed for $2 \mathrm{~h}$ at a temperature of $650^{\circ} \mathrm{C}$ in a $10^{-5}$ mbar vacuum, in order to obtain a structure with a mean grain size of about $100 \mu \mathrm{m}$. Annealed samples were deformed in pure rolling and tension, in order to study the microstructural features developed during these paths and to obtain the stress-strain curve under tension. Samples also underwent the rolling-tension sequence. Pure tension and tension after prestrain were always transverse to the initial rolling direction of the sheet. Two different types of strain path change were studied: the tensile and rolling directions were either parallel or normal to each other. In order to obtain mechanical characterization during the second path under tension, annealed samples were prestrained by rolling up to $0.04,0.20$ and 0.30 von Mises equivalent strain values (the von Mises equivalent strain by rolling is: $\varepsilon=(2 / \sqrt{3})\left|\varepsilon_{3}\right|$, where $\varepsilon_{3}$ is the logarithmic plastic strain in the thickness direction). Other prestrain values by rolling were also used for microstructural characterization. During the second path under tension, microstructural observations were performed at the beginning of deformation (strain value of about 0.025 ) and after rupture. The rolling deformation was always performed on $100 \mathrm{~mm} \times 100 \mathrm{~mm}$ specimens. The tensile samples had a gauge size of $60 \mathrm{~mm} \times 10 \mathrm{~mm}$. All tensile tests were performed at room temperature and with an initial strain rate of $10^{-4} \mathrm{~s}^{-1}$.

The dislocation and twinning microstructure was observed by TEM. Samples for TEM were polished mechanically from both sides down to a thickness of about $0.15 \mathrm{~mm}$ and then electropolished using a double jet thinner. A dilute solution of orthophosphoric acid $(2: 1)$ at room temperature under $10 \mathrm{~V}$ tension was used. TEM analysis was carried out at $100 \mathrm{kV}$.

\section{Results}

\subsection{Mechanical behavior}

Fig. 1 shows the true stress $(\sigma)$-true strain $(\varepsilon)$ curves for pure tension and for tension after rolling at three prestrain amounts $\varepsilon_{\mathrm{p}}=0.04,0.20$ and 0.30 , for both parallel and normal cases of the tensile direction with respect to the rolling direction. For the rolling prestrain value $\varepsilon_{\mathrm{p}}=0.04$, the reloading yield stress, defined as the back extrapolated stress $\left(\sigma_{\text {be }}\right)$ [25], is greater than the reference stress $\left(\sigma_{\mathrm{r}}\right)$ reached at equivalent strain under tension without prestrain, whatever normal or parallel sequence is considered. The value of reloading yield stress $\sigma_{\text {be }}$ is lower than $\sigma_{\mathrm{r}}$ for $\varepsilon_{\mathrm{p}}=0.20$ and 0.30 . The residual uniform strain $\left(\varepsilon_{\mathrm{r}}\right)$, i.e. the strain up to necking during the second path under tension, makes the value of the total deformation before necking $\left(\varepsilon=\varepsilon_{\mathrm{p}}+\varepsilon_{\mathrm{r}}\right)$ greater than the correspondent value obtained under tension without prestrain. The path change increases the total deformation before necking $(\varepsilon \approx 0.47-0.53)$ in comparison to the one for pure tension $(\varepsilon \approx 0.40)$, independently of tensile axis direction in respect to rolling direction.

The evolution of the work-hardening rate $\theta=\mathrm{d} \sigma / \mathrm{d} \varepsilon$ as a function of the true stress $\sigma$ is shown in Fig. 2, both for pure tension and for normal and parallel tension after prestrain in rolling. After an initial relatively high decrease in the work-hardening rate, the pure tension curve reaches an almost linear region, with a small decrease in the work-hardening rate, in the stress range 200 to $290 \mathrm{MPa}$. This is followed by a change of concavity, consistent with the stage B, typical of materials with low SFE and low-to-medium grain size [26]. The reloading curves, at the end of the relatively high initial drop of the work-hardening rate, intersect the one without prestrain, after which the levels of the reloading curves remain lower than the pure tension one up to the necking point, when all reloading curves tend to cross the reference curve again. Different trends are observed for the reloading curves depending on the prestrain

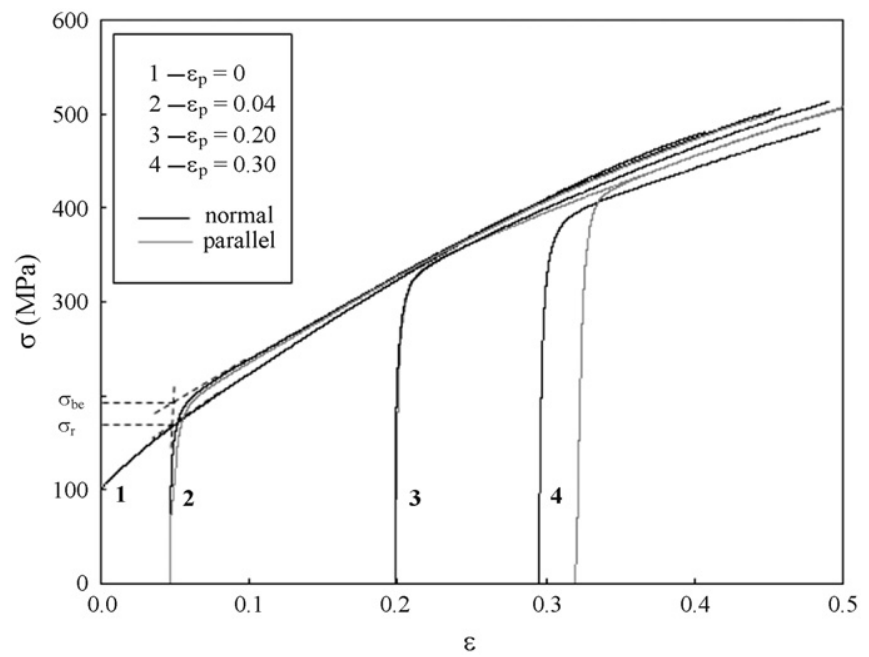

Fig. 1. True stress $(\sigma)$-true strain $(\varepsilon)$ curves in tension for brass samples with and without prestrain in rolling. 


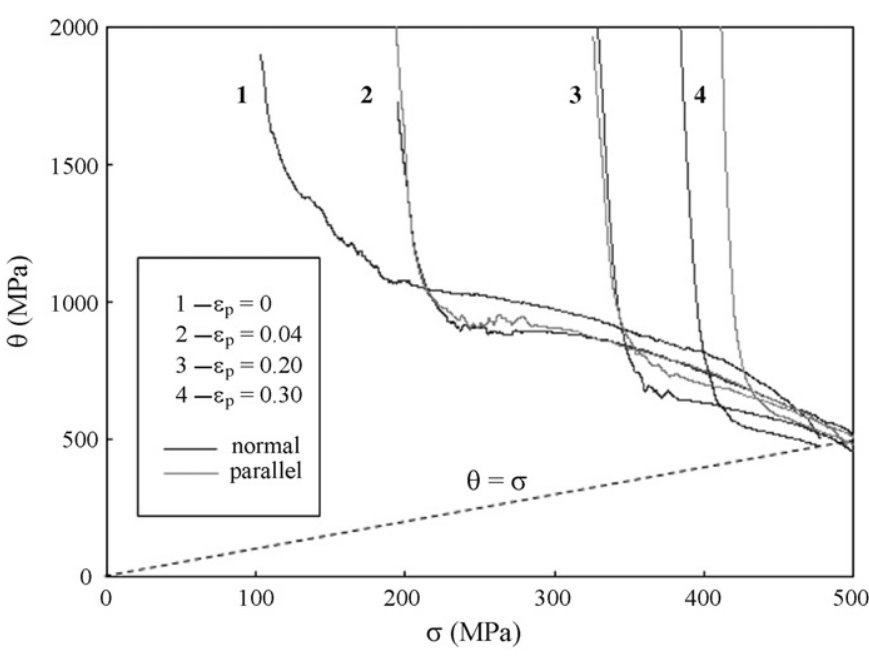

Fig. 2. Work-hardening rate $\theta=\mathrm{d} \sigma / \mathrm{d} \varepsilon$ versus tensile stress $\sigma$ for brass samples with and without prestrain in rolling.

value: for $\varepsilon_{\mathrm{p}}=0.04$ a region with constant work-hardening rate is observed in the stress range mentioned above (200-290 MPa), for which a low decrease in the work-hardening rate under pure tension is observed; for higher prestrain values, $\varepsilon_{\mathrm{p}}=0.20$ and 30 , the curves start at a stress value higher than $290 \mathrm{MPa}$ and the region with a constant work-hardening rate is not observed.

\subsection{TEM observations}

Dislocation and twin microstructure observations were performed using TEM during the direct paths, rolling and tension, and during reloading under tension after parallel and normal rolling. It is worth noting that the existence of twins in all samples observed was confirmed by characteristic twin reflections in diffraction patterns. For clarity, Table 1 summarizes the results related to the presence and morphology of twins observed in simple (pure tension and pure rolling) and complex (tension parallel and normal to previous rolling) strain paths. These results and the results concerning the dislocation microstructures are detailed below.

\subsubsection{Simple strain paths}

Firstly, the dislocation and twin microstructure developed during pure rolling will be analyzed. TEM observations were carried out in brass samples deformed by rolling at $0.04,0.15,0.25$ and 0.50 equivalent strains. When the strain value was 0.04 , tangled low-density dislocation structures were observed. Extended dislocations are present at this strain value (Fig. 3). As the strain value increases up to 0.15 the dislocation structures developed remain similar to the ones at 0.04 equivalent strain by rolling: disordered dislocation structures still predominate. However, more organized dislocation structures are also observed in some regions. For an equivalent strain value of 0.25 , extended dislocations become rare, the dislocation structures are denser and isolated twins and

Table 1

Presence and morphology of twins observed in simple (pure tension and pure rolling) and complex (tension parallel and normal to previous rolling) strain paths.

\begin{tabular}{|c|c|c|c|c|}
\hline & \multicolumn{4}{|c|}{ Simple strain paths } \\
\hline & Strain value & \multicolumn{3}{|c|}{ Occurrence and morphology of twins } \\
\hline Pure rolling & $\begin{array}{l}0.04 \\
0.15 \\
0.25 \\
0.50\end{array}$ & $\begin{array}{l}\text { Absence o } \\
\text { Absence o } \\
\text { Isolated tv } \\
\text { One family } \\
\text { structures }\end{array}$ & $\begin{array}{l}\text { ins } \\
\text { ins } \\
\text { and groups of parallel twins, i } \\
\text { twins in some grains, and also }\end{array}$ & $\begin{array}{l}\text { ome grains } \\
\text { o twin families, intersecting each other in the same grain; all studied grains contain twin }\end{array}$ \\
\hline \multirow[t]{3}{*}{ Pure tension } & $\begin{array}{l}0.04 \\
0.10 \\
0.25 \\
0.50\end{array}$ & \multicolumn{3}{|c|}{$\begin{array}{l}\text { Individual twins of one twin family in some grains } \\
\text { A significant number of deformation twins both individual and groups of parallel twins; ample amount of grains are free from } \\
\text { deformation twinning }\end{array}$} \\
\hline & & & \multicolumn{2}{|l|}{ Complex strain paths } \\
\hline & & & Prestrain value in rolling $\left(\varepsilon_{\mathrm{p}}\right)$ & Occurrence and morphology of twins \\
\hline \multicolumn{3}{|c|}{ Tension $(\varepsilon=0.025$ ) after normal rolling } & $\begin{array}{l}0.04 \\
0.20 \\
0.30\end{array}$ & $\begin{array}{l}\text { Individual twins and one family of parallel twins in a few grains } \\
\text { Individual twins and one family of parallel twins in a few grains } \\
\text { One twin family and twin intersections, i.e. two families of parallel twins in the same } \\
\text { grain; amount of twins grows }\end{array}$ \\
\hline \multicolumn{3}{|c|}{ Tension $(\varepsilon=0.025)$ after parallel rolling } & $\begin{array}{l}0.04 \\
0.20 \\
0.30\end{array}$ & $\begin{array}{l}\text { Individual twins and one twin family; twins are morphologically identical to those } \\
\text { developed at the normal tension } \\
\text { Individual twins one and family of parallel twins } \\
\text { One twin family and also two families of parallel twins in the same grain; second twin } \\
\text { system occurs less frequently than when tension is normal to rolling }\end{array}$ \\
\hline \multirow{2}{*}{\multicolumn{3}{|c|}{ Tension (up to rupture) after normal rolling }} & 0.04 & $\begin{array}{l}\text { A large number of twins of one family; in some grains, twins of two intersecting families; } \\
\text { the density of twins is higher than in cases of monotonic strain paths, deformed up to } \\
\text { rupture }\end{array}$ \\
\hline & & & 0.20 & $\begin{array}{l}\text { Twin microstructure is qualitatively identical to the microstructure for the lower } \\
\text { prestrain value } 0.04 \text {, but intersections of two twin families occur more often } \\
\text { Twin microstructure is qualitatively identical to the microstructure for the lower } \\
\text { prestrain value } 0.20\end{array}$ \\
\hline \multirow{2}{*}{\multicolumn{3}{|c|}{ Tension (up to rupture) after parallel rolling }} & $\begin{array}{l}0.04 \\
0.20\end{array}$ & $\begin{array}{l}\text { A large number of twins of one family; in some grains, twins of two intersecting families } \\
\text { Twin microstructure is qualitatively identical to the microstructure for the lower } \\
\text { prestrain value } 0.04\end{array}$ \\
\hline & & & 0.30 & $\begin{array}{l}\text { Twin microstructure is qualitatively identical to the microstructure for the lower } \\
\text { prestrain value } 0.20 \text {; the second twin system occurs less frequently than when tension is } \\
\text { normal to rolling }\end{array}$ \\
\hline
\end{tabular}




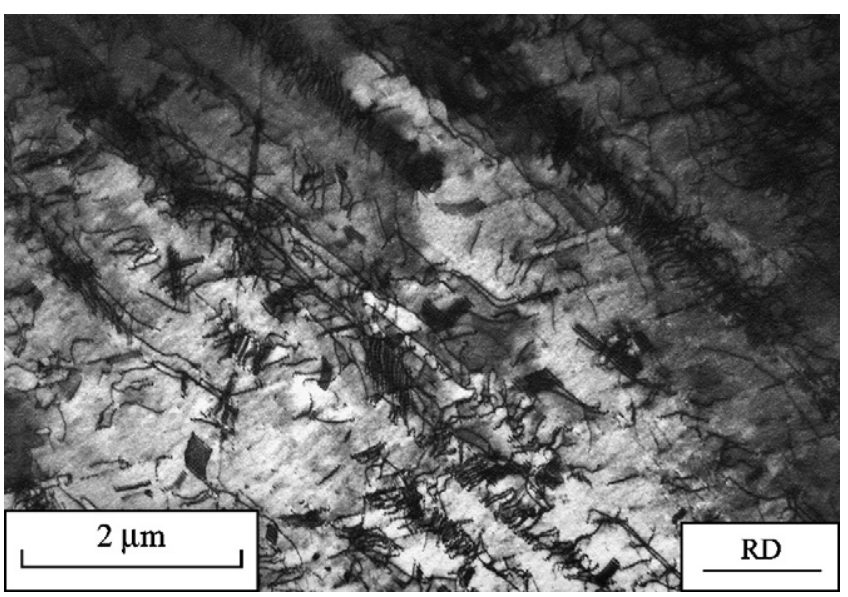

Fig. 3. Extended dislocations developed in brass after a rolling equivalent strain $\varepsilon=0.04$ (RD: rolling direction).

groups of parallel twins are present, in some grains. In brass samples deformed up to an equivalent strain value of 0.50 , the twin structures are commonly observed: all studied grains contain twin structures. One family of twins appears in some grains, but two twin families were also observed, intersecting each other in the same grain, as shown in Fig. 4(a). An example of the diffraction pattern showing the presence of twin reflections is shown in Fig. 4(b), which covers the region of Fig. 4(a).

Strain values of $0.04,0.10,0.25$ and 0.50 were chosen to study dislocation and twin structure evolution during pure tension. TEM observations showed that, for the initial stages of deformation, the developed microstructure during pure tension is basically identical to one observed in pure rolling. After a tension strain value of 0.04 , disordered dislocation structures and significant amount of extended dislocations were observed (Fig. 5). The extended dislocations are a more frequent structural feature than those formed by rolling at the same strain value. At a strain value of 0.10 , the dislocation microstructure is essentially similar to the one developed at the strain value of 0.04 . Qualitative change in the deformation microstructure was observed for the strain value of 0.25 . Aligned dislocation structures become more organized and dense. Individual twins of one twin family are present and the number of grains with deformation twinning is approximately the same as for equal value of deformation in pure rolling. The samples cut in the necking region of the tensile samples (plastic strain close to 0.50 ) show a significant number of deformation twins: individual as well as groups of parallel twins (Fig. 6); but, even in this case, it is possible to observe grains free from deformation twinning. The deformation twins appear in nearly $50 \%$ of grains examined, whereas they are present in almost all grains studied after deformation by rolling at the same strain level.

\subsubsection{Complex strain paths}

Two types of sequential strain paths were investigated, in order to understand the influence of the change of strain path and the type of change on the mechanical behavior and on the dislocation and twin microstructure developed after reloading. For both cases, the brass was prestrained by rolling up to von Mises equivalent strains of $0.04,0.20$ and 0.30 , after which normal and parallel tension was performed up to two strain values: 0.025 and up to rupture. A study of dislocation and twin structure developing at the initial stages of tensile deformation after prestraining by rolling was carried out in order to establish the microstructural features immediately after strain path change and to better understand work-hardening behavior during complex strain paths.

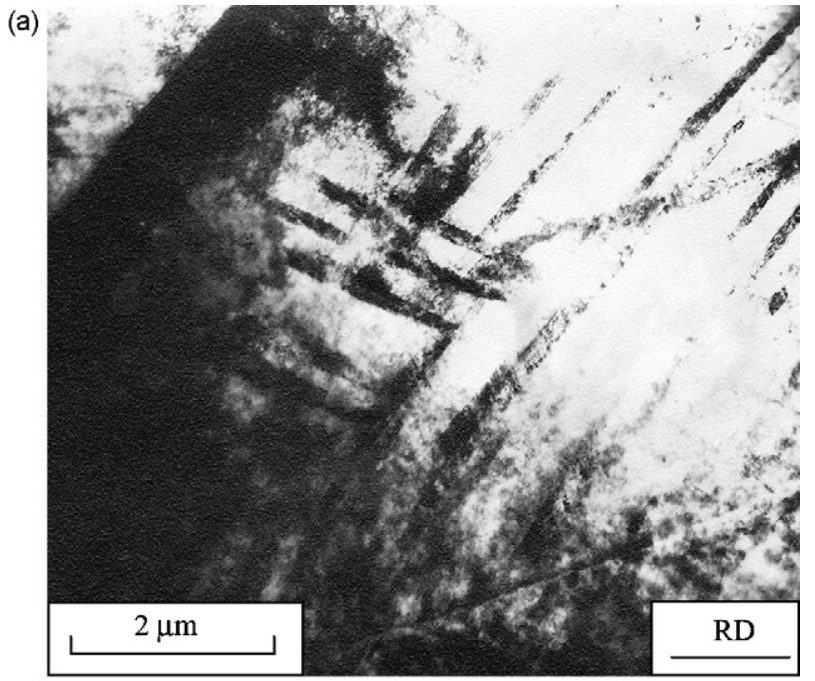

(b)

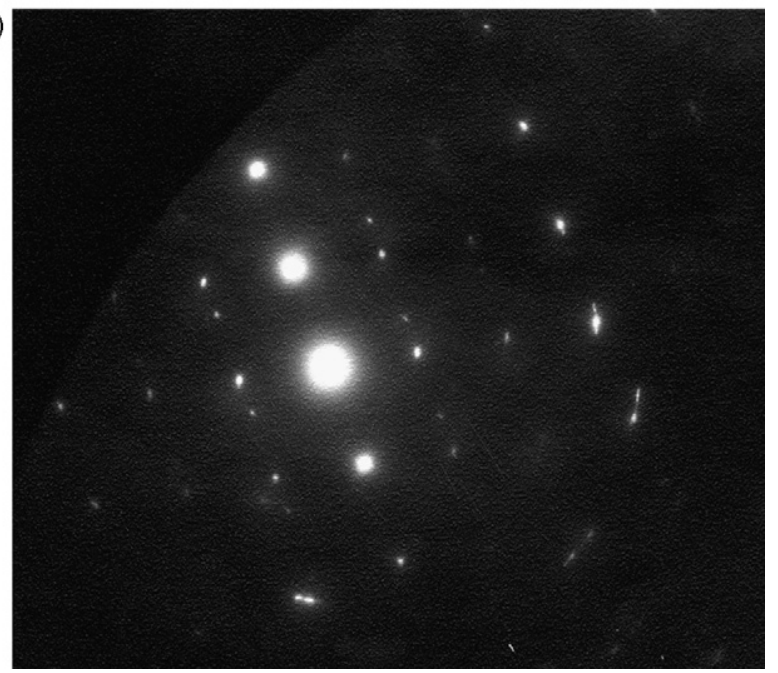

Fig. 4. Twins' intersection (a) developed after a rolling equivalent strain $\varepsilon=0.50$ (RD: rolling direction) and (b) diffraction pattern of the TEM image show in (a).

The evolution of dislocation and twin structure in complex strain paths is significantly different to that in simple ones. Whatever the change of strain path (parallel or normal) and the prestrain by rolling value, twins are become apparent in the second strain path under tension, from the beginning of reloading, i.e. strain of

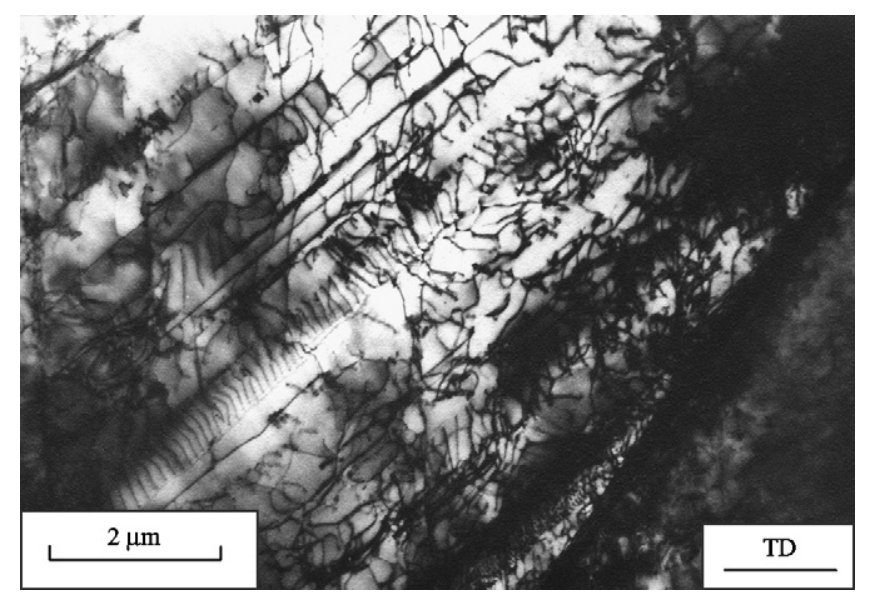

Fig. 5. Unorganized dislocation structures developed in simple tension $\varepsilon=0.04$ (TD: tensile direction). 


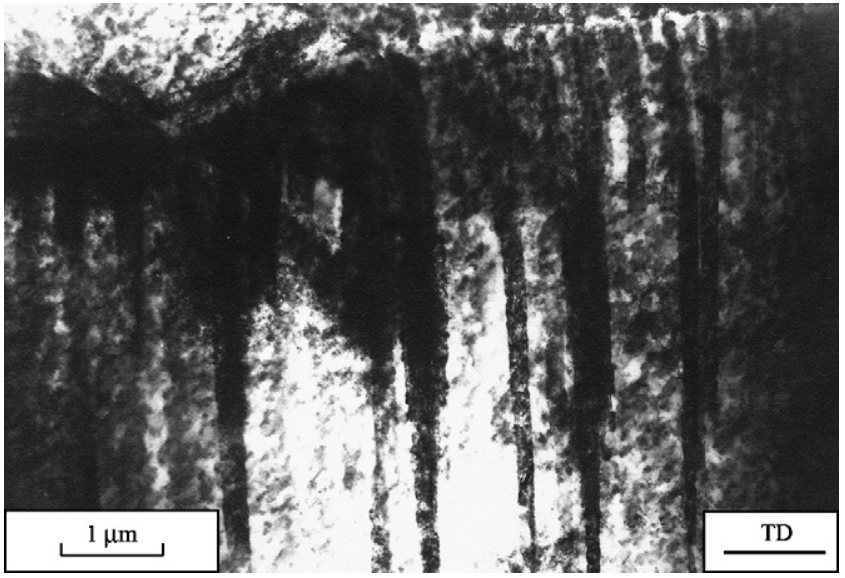

Fig. 6. Deformation twins developed in simple tension $\varepsilon=0.50$ (TD: tensile direction).

0.025. In the case of a lower prestrain value (0.04), the total strain value is 0.065 , and so is lower than that for twinning development under pure tension. However, during the second strain path under tension after prestraining to this value by rolling, individual twins and one family of parallel twins can be seen in a few grains. These twins are morphologically identical to those developed at higher plastic deformation stages, during simple strain paths of rolling and tension. The typical twin microstructure that develops during the initial stage of second strain path is shown in Fig. 7. Tangled dislocation structures containing large amounts of extended dislocations and poorly organized aligned dislocation structures can be seen in some regions, as for low values of pure tensile deformation. Further increasing the prestrain value $\left(\varepsilon_{p}=0.20\right)$ does not lead to any change in microstructural features developed during the early stages of the second path under tension (strain equal to 0.025). Only one family of parallel twins is observed in each grain and dislocation density becomes higher than in the previous prestrain case. For the higher prestrain value $\left(\varepsilon_{\mathrm{p}}=0.30\right)$ the amount of twins grows and twin intersections can appear, i.e. two families of parallel twins were observed in the same grain, for both normal and parallel tension (Fig. 8).

With regard to the samples deformed up to rupture after parallel or normal prestrain, intense twinning was observed in the samples prestrained by rolling up to 0.04 and deformed up to rupture. For these samples, a large number of twins of one family are frequently observed in the grains (Fig. 9). In some grains, twins of two

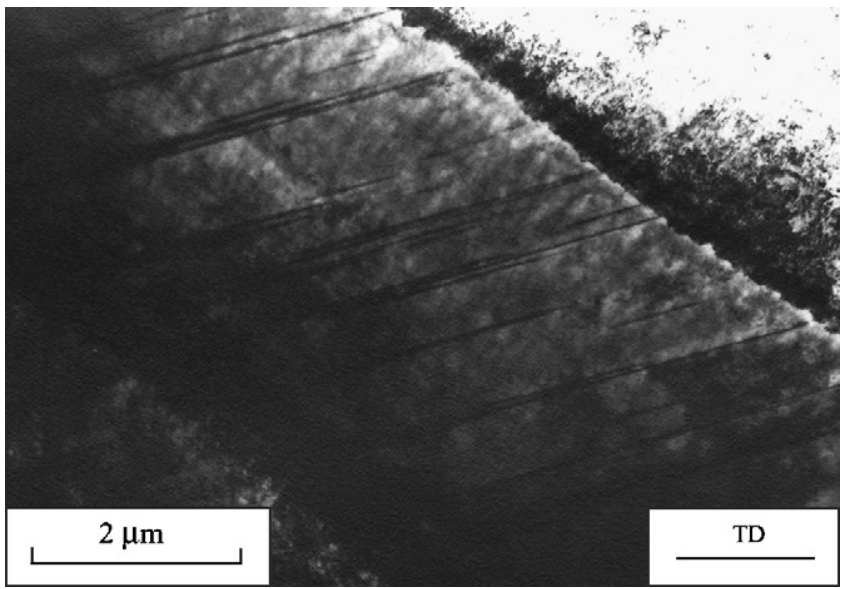

Fig. 7. Deformation twins developed in tension $(\varepsilon=0.025)$ after prestrain in rolling $\left(\varepsilon_{\mathrm{p}}=0.04\right)$ for tension normal to rolling (TD: tensile direction).

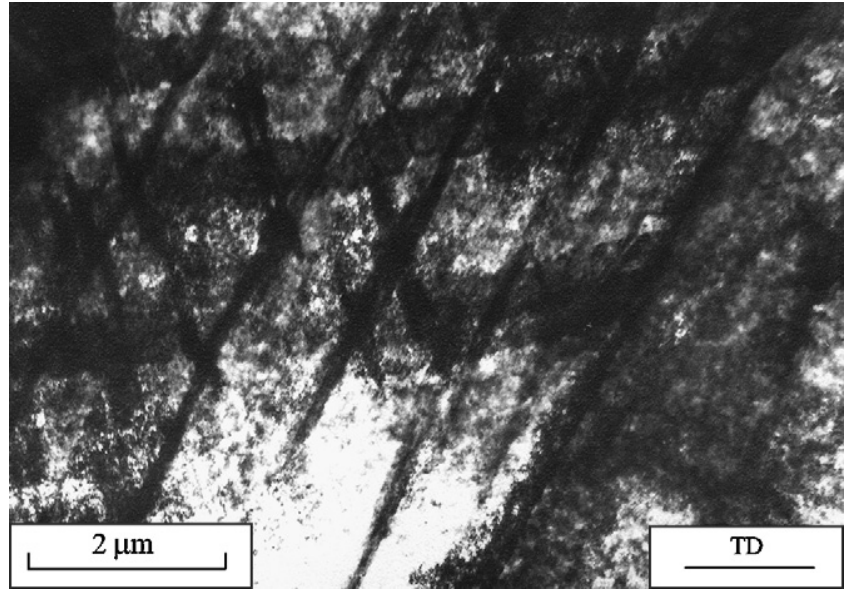

Fig. 8. Twins' intersections developed in tension $(\varepsilon=0.025)$ after prestrain in rolling $\left(\varepsilon_{\mathrm{p}}=0.30\right)$, tension is normal to rolling (TD: tensile direction).

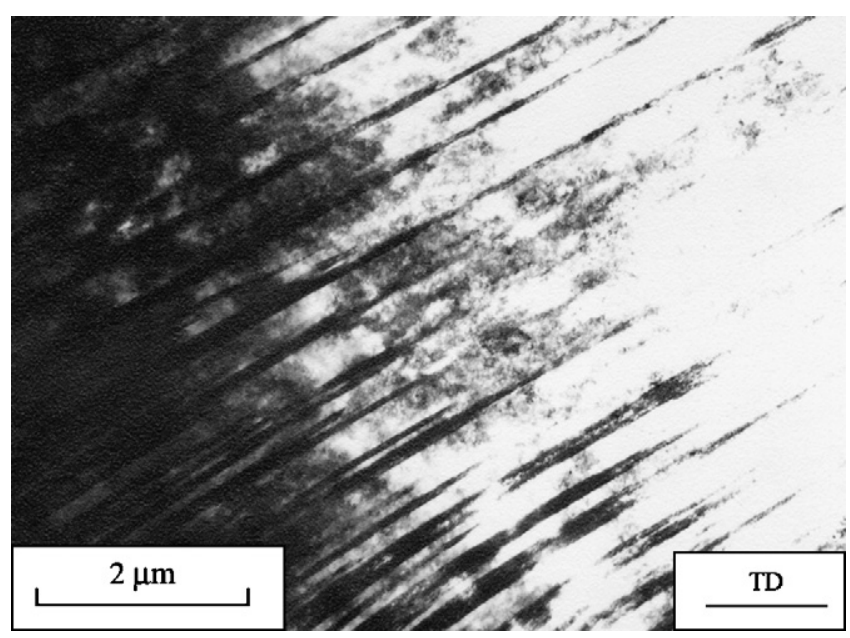

Fig. 9. Deformation twins of one family developed in brass samples deformed in tension up to rupture. The prestrain value in rolling is $\varepsilon_{\mathrm{p}}=0.04$ and the tension is parallel to rolling (TD: tensile direction).

intersected families are present (Fig. 10). The density of twins is higher than in cases of simple strain paths, deformed up to rupture. For samples prestrained up to 0.20 and 0.30 by rolling in the first strain path, the dislocation and twin microstructure observed

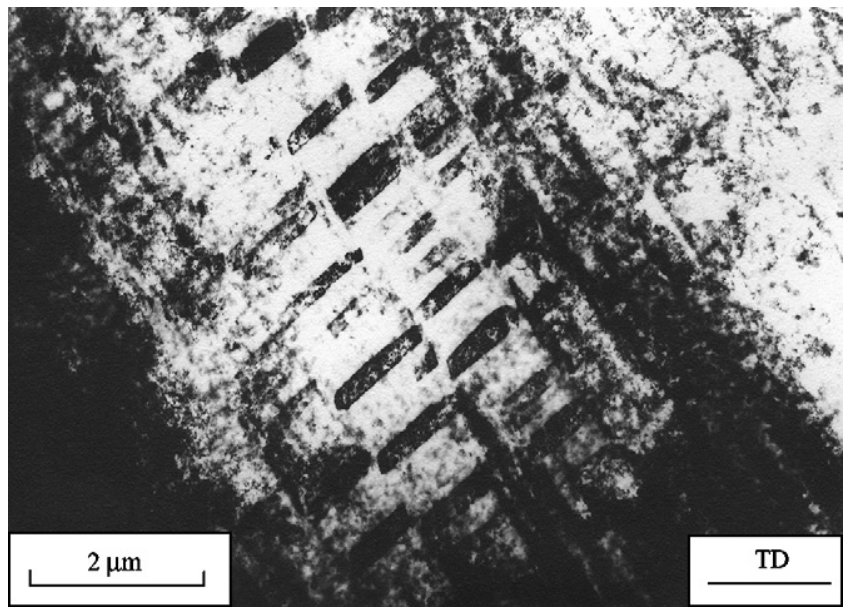

Fig. 10. Twins' intersections developed in tension up to rupture after prestrain in rolling $\left(\varepsilon_{\mathrm{p}}=0.04\right)$; tension is parallel to rolling (TD: tensile direction). 


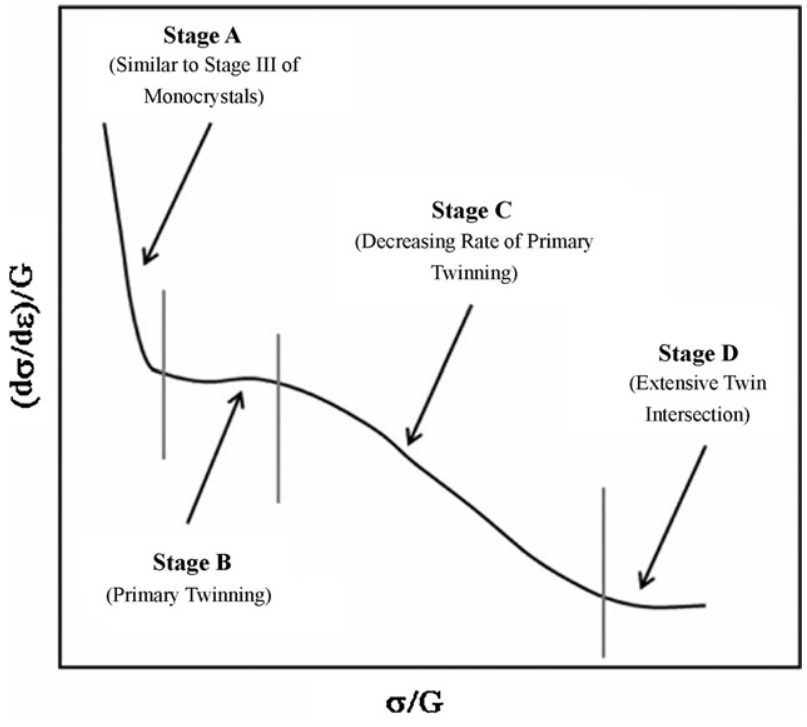

Fig. 11. Schematic representation of the four stages of strain-hardening in FCC materials with low stacking fault energy [27].

after rupture in the second path under tension, is qualitatively identical to the microstructure for the lower prestrain value 0.04 , but intersections of two twin families occur more often.

Generally, the microstructures observed for both complex strain paths are similar, as noted above. However, certain particular microstructural features distinguish both types of strain path. Twins of only one family predominate after rupture, whatever the change of strain path and the prestrain values, but the second twin system occurs less frequently when tension is parallel to rolling than when tension is normal to rolling. This is also observed when the strain value under tension equals 0.025 , for the case where prestrain equals 0.30 . Thus, two intersecting families of twins are more likely after strain path change when tension is normal to rolling.

\section{Discussion}

\subsection{Simple strain paths}

It has been reported that the evolution of the work-hardening rate versus stress of FCC materials with low stacking fault energy presents four stages [23,26-32], as shown schematically in Fig. 11. An initial sharp decrease in the work-hardening rate is observed, so called stage A. This stage, similar to stage III of monocrystals, is usually observed in FCC polycrystals with high stacking fault energy during a relatively large range of plastic deformation obtained by tension. A constant work-hardening rate, stage $B$, follows the initial work-hardening decrease. The onset of stage B was correlated with the initiation of twinning. Stage $C$ is characterized by a renewed decrease in the work-hardening rate. During this stage, the rate of primary twinning decreases. Finally, stage D was correlated with extensive twin intersection, brought about by the activation of a second twinning system non-coplanar with the previous one.

For materials with low-to-moderate stacking fault energy, plastic deformation by twinning usually takes place after a moderate strain value. The activation of deformation twins competes with dislocation slip and affects the mechanical response. El-Danaf et al. [26] and Asgari et al. [27] suggest that the initiation of deformation twinning requires a critical dislocation density, measured by the value $\left(\sigma-\sigma_{0}\right) / G$ (where $\sigma$ is the flow stress during deformation, $\sigma_{0}$ is the initial yield strength of the material and $G$ is the shear modulus). Also, the average homogeneous slip-length controls the deformation by twinning: in materials with low values of
SFE a delay occurs in the development of in-grain misorientations and consequently the slip length remains close to the initial grain size, at least until twinning starts. In these materials, deformed under static mechanical tests at room temperature, the microstructural variables promoting twinning are a combination of a relatively high dislocation density with relatively high average slip length, i.e. large homogeneous deformation-length scale $[26,27]$. The evolution of the dislocation density and the average slip length with strain depends on SFE, but also on other material variables such as the grain size [26].

The strengthening observed during stage $B$ has been attributed to two different physical mechanisms. The most common explanation is the reduction of free slip line distance due to the presence of twins [23,26,28-32]. This is similar to the Hall-Petch effect: the twin-matrix interface acts as barrier to dislocation pile-up formation and the strain accommodation is more complex due to the reduction of the mean slip length. Other authors [33] consider that, as a result of twinning shear transformation, some glissile dislocations are converted into sessile configurations and other dislocations become less glissile after twinning. Whatever the case, the consequence is identical: the twinning development leads to a work-hardening rate higher than that measured in the absence of twinning.

The results of the current study show that stage B is noticeable for the case of tension without prestrain (Fig. 2). However, the work-hardening rate versus stress curve shows a feature different from that usually observed for materials with higher SFE [5] and rather similar to that usual for materials with low SFE, with relatively low grain size [26]. After the initial sharp decrease in the work-hardening rate, the curve follows a linear region with almost constant work-hardening rate (between $200(\varepsilon=0.08)$ and $290 \mathrm{MPa}$ $(\varepsilon=0.165))$, which is consistent with the stage B [26]. These stress values correspond to $\left(\sigma-\sigma_{0}\right) / G=0.0025$ and 0.0050 , respectively, which are quite similar to the ones previously found for the beginning and the end of stage B in $70 / 30$ brass with 30 and $250 \mu \mathrm{m}$ grain size [26]. Stage B of work-hardening can be more or less discernible, depending on the grain size. With decreasing the grain size, this stage tends to disappear, which was explained by the relatively small extension of the twin clusters during this stage, for low and medium grain-size material, i.e. fewer deformation twins are produced for low and medium grain-size than for higher grain sizes materials [26]. In this study, of $70 / 30$ brass with $100 \mu \mathrm{m}$, the evolution of twin microstructure also occurs gradually with deformation, as testified by our TEM observations, which justifies the work-hardening behavior observed, showing a perceptible stage B.

The dislocation structure presented above does not show clear organization of dislocations in cell structures that means extremely modest dynamic recovery during deformation. The organization of the dislocations in well defined dislocation cells, as observed in materials with high SFE, for example, copper [4,5], aluminum [9] or mild steel [3], requires easy cross-slip, which has a low probability of occurring in materials with low SFE, such as the 70/30 brass studied here. The activation of two, eventually three, main slip systems in most grains is observed under tension and rolling from the beginning of deformation. This means that the strain compatibility between adjacent grains is provided by geometrical dislocations, whose effects are observed all over the grain and not just close to the grain boundaries. This is in agreement with the medium grain size $(100 \mu \mathrm{m})$ of the studied $70 / 30$ brass sheet and indicates that the grain deformation follows a slip pattern, indicative of a concurrence between the Sachs (just one slip system) and the Taylor model (three or more main slip systems) as previously observed for copper [4].

From current experimental results, it was possible to conclude that deformation in only one twinning system seems to be favorable which agrees with the above discussion concerning the mechani- 
cal behavior. In fact, when two twinning systems are observed in the same grain they are restricted to different regions (the internal stress due to the accommodation process between adjacent grains may induce distinct twinning systems in different regions, particularly if the activities of the systems are similar). The absence of grains with multiple twinning in the same region has been justified by a reciprocal hardening of twinning systems (similar to latent hardening of slip systems), i.e. the critical resolved shear stress necessary to activate one twinning system should be lower than the one necessary to activate more than one twinning system [26,27]. The activation of just one twinning system during the relatively low deformation range studied is in agreement with the results of El-Danaf et al. [26] for stage B.

After stage $B$, the work-hardening rate versus stress shows a clear decrease in the work-hardening rate. This is the so-called stage $\mathrm{C}$, with decreasing rate of primary twinning. In fact, our TEM observations do not show much of twin density up to a deformation value of about 0.25 . Also, it was observed that just one twin system occurs during this stage for pure tension up to rupture (strain value higher than 0.40 ). Deformation by only one twin system occurs because the critical resolved shear stress is lower than the one necessary for activation of multiple twinning. In fact, previous results by ElDanaf et al. [26] show that stage D (corresponding to extensive twin intersections) is initiated in $70 / 30$ brass with 30 and $250 \mu \mathrm{m}$ grain sizes when $\left(\sigma-\sigma_{0}\right) / G$ approached a value of about 0.015 (obtained in simple compression), which corresponds to a stress values of $670 \mathrm{MPa}$, slightly higher than the maximum stress value attained under tension. This signifies that the levels of stress which need for a new twin to shear through an existing twin are not reached in pure tension.

The TEM observations after rolling essentially confirm the microstructural results observed under tension: up to an equivalent von Mises deformation equal to 0.10 , twins are not observed and typically just one family of twins was observed for moderate strain values ( 0.15 and 0.25 ). However, for severe deformation by rolling (equivalent von Mises deformation equal to 0.50 ), two twin families appear in some grains, intersecting each other, which does not occur under tension. The appearance of two twin families in pure rolling indicates that, the stress attained in this strain path at an equivalent von Mises deformation of 0.50 , is certainly higher than that achieved under tension for the maximum strain value (0.40). Actually, pure rolling deformation is more constrained (the strain path is imposed and the grains cannot deform in the transverse direction) than tension (the stress path is imposed) and requires a larger number of active systems for most grain orientations, independently of the accommodation between grains [6].

\subsection{Complex strain paths}

Two different types of mechanical behavior were observed after path change. For a low prestrain value (at about 0.04 ), the macroscopic reloading yield stress is higher than the stress reached on the tension curve without prestrain, at a strain which is equal to the prestrain amount. For the higher prestrain values ( 0.20 and 0.30 ), the value of the back extrapolated stress is lower than the reference stress. In this context, the case of low prestrain value is firstly analyzed and, afterwards, the two other cases of prestrain values are examined.

\subsubsection{Low prestrain value}

The increase in yield stress after path change has been analyzed in previous work for materials with moderate-to-high SFE $[5,6]$ and was related to the physical mechanisms, i.e. the latent hardening effect that occur during reloading. Using Sachs and Taylor models, a statistical analysis of the sets of active slip systems during each path, has shown that, in most of the grains of FCC metals under tension normal to previous rolling, the active slip systems during reloading under tension were not activated during the first rolling path [8]. Also, in some grains under tension after parallel rolling, activation of new slip systems not active during prestrain in rolling must be expected, but the number of new active slip systems is inferior to that found under tension after normal rolling. So, for the low prestrain value (0.04), the observed increase in yield stress of brass under tension normal and parallel to previous rolling can also be explained by the interaction between the newly active slip systems, developed under tension, and the ones active during rolling. This is usually called the latent hardening effect and agrees with the facts that the increase in yield stress is higher under tension normal to rolling than parallel to it (see Fig. 1) and no twins were observed for such low prestrain value in rolling.

From our TEM observations it was not possible to identify the dissolution of the dislocation microstructure under tension both normal and parallel to previous rolling, which is usually observed in materials with high SFE [3,5]. This is due to the inhibition of dynamic recovery mechanisms: as in other metals with low SFE, small amounts of climb, cross-slip and rearrangement of dislocations take place in brass. As a consequence of the lack of dynamic recovery mechanisms, there is no significant decrease in the total forest dislocation density after path change. In this way, the relatively high value of the back extrapolated stress $\left(\sigma_{\text {be }} / \sigma_{\mathrm{r}} \approx 1.07\right.$ for tension normal to rolling and $\sigma_{\mathrm{be}} / \sigma_{\mathrm{r}} \approx 1.03$ for tension parallel to rolling) due to latent hardening effects observed after path change (Fig. 1) is not followed by a continuous decrease in the work-hardening rate, as is usually observed for high SFE materials after low prestrain values (as shown in the example of Fig. 12 [36]). However, this is not the only cause that explains this mechanical behavior, shown in Fig. 2. A second cause is twin development, although this is related to the first, as discussed following.

With regard to the work-hardening rate evolution after path change, the curves $\theta$ versus $\sigma$ exhibit, for the low prestrain value (at about 0.04 ), behavior different to that usually observed for material with higher stacking fault energy, such as mild steel [3] and copper $[5,6]$. In fact, for these materials, the curves after low values of prestrain cross the one without prestrain and after the cross-point they always decrease up to necking (Fig. 12). This is due to the intensity of recovery in reloading, as established by observations of the dissolution of the prior microstructure after path change. This is not the case with these results for $70 / 30$ brass; the work-hardening

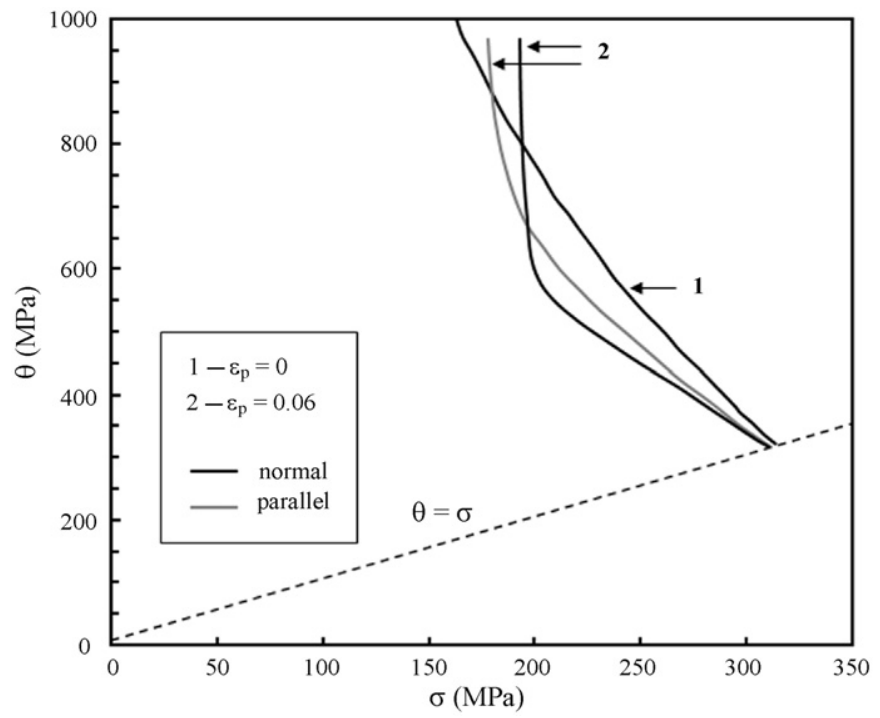

Fig. 12. Work-hardening rate $\theta=\mathrm{d} \sigma / \mathrm{d} \varepsilon$ versus tensile stress $\sigma$ for copper samples with and without prestrain in rolling $\left(\varepsilon_{\mathrm{p}}=0.06\right)[36]$. 
rate evolution after path change shows an initial decrease (as for materials with higher stacking fault energy), but this is followed by a transient region with constant work-hardening rate, which must be related to the beginning of twin formation, i.e. with stage B in simple tension. This is confirmed by TEM results that show that at a strain value of 0.025 in reloading (total strain value of 0.065 ), one family of twins is already present; in pure tension twin activity is detected only for $\varepsilon$ higher than 0.10 . In fact, the path change causes the stress estimated above for the beginning of stage $B$ under pure tension (at about $200 \mathrm{MPa}$ ) to be overcome from the beginning of tension after normal or parallel rolling. As under pure tension, the region of constant work-hardening rate ends with a stress of $\left.290 \mathrm{MPa}\left(\sigma-\sigma_{0}\right) / G=0.0050\right)$, as mentioned above. Also, the value of $900 \mathrm{MPa}$ for the constant work-hardening rate $(\theta / G=0.024)$ is close to the one for simple tension and within the range found in the literature of about $0.02-0.03 \mathrm{G}$ for $70 / 30$ brass and other materials [26]. The stress region with a constant work-hardening rate is similar for both types of strain paths: tension after normal or parallel rolling. Only a small difference (less than $50 \mathrm{MPa}$ ) is observed in the constant work-hardening region between these two types of strain paths: the work-hardening rate is higher for the case of tension after parallel rolling. The slightly lower level of stage B in the case of normal path change can be explained by a slightly more intense dynamic dislocation recovery mechanism for normal path change than for parallel path change, owing to the more drastic change of slip systems.

In conclusion, twin development under tension after rolling, at equivalent total strain values lower than the strain observed for twinning onset during pure rolling and pure tension, is related to the fact that the activation of deformation twinning competes with dislocation slip. After path change, the latent hardening effect increases the critical resolved shear stress necessary for dislocation slip and, consequently, the critical resolved shear stresses for twinning and for slip converge, which is necessary for twinning activity. Moreover, the appearance of the twins immediately after path change, also contributes to the decreased work-hardening rate, and leads to the appearance of a clear stage B under tension after rolling.

\subsubsection{High prestrain values}

According to the TEM observations, the deformation twinning in brass samples prestrained by rolling begins immediately after strain path change and twin microstructure is already well-developed at the initial stages of tensile deformation $(\varepsilon=0.025)$ whatever prestrain value during first strain path is considered. The twinning intensity increases with the increase in the value of prestrain rolling, becoming significant for the cases of rolling prestrain equal to 0.20 and 0.30 , where some twin structures have been formerly developed during first strain path and new twin systems can interact with them to form twin intersections.

For the higher prestrain values ( 0.20 and 0.30$)$, the back extrapolated stress is lower than the reference stress, as has already been observed by other authors [34,35]. This indicates that the effects of latent hardening do not control the mechanical behavior. In fact, the TEM observations show that, for these prestrain values, twins are already present at the end of the prestrain path by rolling and so stage B was attained. After path change, the new active dislocation slip systems have a different orientation in relation to the twin plane, which increases the mean slip length of the new active dislocations and leads to a consequent reduction in the pile-up stress in these dislocations, at the beginning of reloading. This was confirmed by a simple calculation of the Schmid factor for dislocation slip and twinning systems of several grain orientations, during the prestrain path, that allows the active slip and twinning systems (with the highest Schmid factors) to be determined. Afterwards, for each correspondent grain orientation, the Schmid factors were calculated for the dislocation slip systems under normal tension. The results show that, in general, the orientation of the new active slip systems under tension is such that the mean slip length, controlled by the presence of twins, increases during subsequent tension, when compared with the mean slip length of the active slip systems after rolling. This can explain the results in Fig. 1, which shows that $\sigma_{\text {be }} / \sigma_{\mathrm{r}}$ is lower than 1 for high prestrain values ( 0.20 and $0.30)$.

With regard to the work-hardening rate evolution after high prestrain values, the $\theta$ versus $\sigma$ curves present an initial sharp decrease, after which they decrease slowly until rupture, remaining relatively close to the values found for simple tension. In contrast to the case of low prestrain value, in this case the $\theta$ versus $\sigma$ curves do not contain a transient region with a constant work-hardening rate, typical of stage B. In fact, twins develop during the first path by rolling and stage $C$ occurs at the beginning of the second path, under tension. As a consequence of the relatively high level of the work-hardening rate after path change (i.e. clearly above the necking line: $\theta=\sigma$ ), the total deformation value up to necking is higher in complex strain paths (rolling followed by tension) than in simple tension.

Microstructural observations showed two twinning noncoplanar systems under tension up to rupture subsequent to prestrain rolling, for any prestrain value and path change. Also, in the case of prestrain by rolling at 0.30 , two twin systems were observed for a low strain value of deformation under tension (0.025). The change in the orientation of the grains in relationship to the tensile axis produce an increase in the effective shear stress in new twinning systems that become active, and a second family of twins develops after path change. However, the constant region of work-hardening rate was not observed after path change on $\theta$ versus $\sigma$ curves (Fig. 2 ), in the cases of higher prestrain levels $(0.20$ and 0.30 ), for which twins were already present at the end of the first path in rolling. This must be related to the fact that two opposite mechanisms are present after path change: (i) the dislocation structure becomes unstable, which increases recovery due to dislocation annihilation (this recovery becomes stronger with increasing prestrain value $[4,5]$ ) and (ii) the twinning development, which leads to a work-hardening rate higher than that measured in the absence of twinning.

\section{Conclusions}

Mechanical behavior and microstructural evolution were studied in brass sheets with an average grain size of $100 \mu \mathrm{m}$ subjected to tension, rolling and tension after rolling deformation sequences, tension being normal or parallel to rolling. Twinning appears during simple strain paths under tension and rolling at an equivalent strain higher than 0.10. With increasing strain the twinning becomes intensive in the case of simple rolling, whereas for simple tension the twin amount is lower and volume fraction containing twins is smaller. Moreover, considering only simple strain paths, a second twin family is only observed for large plastic deformation (more than 0.50 ) in rolling. The microstructural observations for simple tension are confirmed by the work-hardening versus stress curve, showing a perceptible stage B connected with deformation twins, which is usually observed for low SFE metals.

Deformation twinning develops during complex strain path for both strain path change directions, parallel and normal. One family of twins appears immediately after strain path change and then two twin systems were observed with increasing deformation up to rupture, although twins intersections are rarer in parallel tension then in normal tension. The twinning structures mainly arise during the second path under tension, and so the strain path change is an effective way to activate deformation twinning. Twinning is more intensive and volume fraction of twins is larger under tension after 
strain path change than for pure tensile deformation where multiple twinning was not observed at all. The work-hardening behavior during a complex strain path for a low prestrain value $(0.04)$ is connected with the dislocation latent hardening effect, whereas for high prestrain values $(0.20$ and 0.30$)$ the mechanical behavior is also related to twin-matrix interface strengthening.

\section{Acknowledgement}

One of the authors, N.A. Sakharova, was supported by a grant for scientific research from the Portuguese Science and Technology Foundation. This support is gratefully acknowledged.

\section{References}

[1] S. Ikeda, Jpn. J. Appl. Phys. 11 (1972) 1273-1283.

[2] Y. Kawasaki, T. Takeuchi, Scr. Metall. 14 (1980) 183-188.

[3] J.V. Fernandes, J.H. Schmitt, Phil. Mag. A 48 (1983) 841-870.

[4] J.J. Gracio, J.V. Fernandes, J.H. Schmitt, Mater. Sci. Eng. A 118 (1989) 97-105.

[5] J.H. Schmitt, J.V. Fernandes, J.J. Grácio, M.F. Vieira, Mater. Sci. Eng. A 147 (1991) 143-154.

[6] J.V. Fernandes, M.F. Vieira, Metall. Mater. Trans. A 28A (1997) 1169-1179.

[7] M.F. Vieira, J.V. Fernandes, J. Mater. Process. Technol. 47 (1995) 261-272.

[8] M.F. Vieira, J.V. Fernandes, B. Chaparro, Mater. Sci. Eng. A 284 (2000) 64-69.

[9] F. Barlat, J.M.F. Duarte, J.J. Gracio, A.B. Lopes, E.F. Rauch, Int. J. Plasticity 19(2003) $1215-1244$

[10] W.B. Hutchinson, R. Arthey, P. Malmstrom, Scr. Metall. 10 (1976) 673-675.

[11] J.H. Schmitt, E. Aernoudt, B. Baudelet, Mater. Sci. Eng. 75 (1985) 13-20.

[12] J.W. Christian, S. Mahajan, Prog. Mater. Sci. 39 (1995) 1-157.
[13] W.S. Lee, C.F. Lin, Metall. Mater. Trans. A 33A (2002) 2801-2810.

[14] B.M. Chaparro, M.M. Vieira, J.V. Fernandes, Mater. Sci. Forum 455-6 (2004) 711-716.

[15] B.M. Chaparro, J.V. Fernandes, Key Eng. Mater. 230-2 (2002) 509-512.

[16] M.M. Vieira, B.M. Chaparro, M.F. Vieira, J.V. Fernandes, Mater. Sci. Forum 455-6 (2004) 280-284.

[17] N.A. Sakharova, J.V. Fernandes, Mater. Chem. Phys. 98 (2006) 44-50.

[18] S.A. Quinones, L.E. Murr, Phys. Stat. Sol. (a) 166 (1998) 763-789.

[19] L.E. Murr, E.A. Trillo, A.A. Bujanda, N.E. Martinez, Acta Mater. 50 (2002) 121-131.

[20] L.E. Murr, E.V. Esquivel, J. Mater. Sci. 39 (2004) 1153-1168.

[21] E.C.S. Correa, M.T.P. Aguilar, E.M.P. Silva, P.R. Cetlin, J. Mater. Process. Technol. 142 (2003) 282-288.

[22] E.C.S. Correa, M.T.P. Aguilar, P.R. Cetlin, J. Mater. Process. Technol. 124 (2002) 384-388.

[23] E. El-Danaf, S.R. Kalidindi, R.D. Doherty, Int. J. Plasticity 17 (2001) 1245-1265.

[24] K.P. Rao, E.V.R.K. Mohan, Key Eng. Mater. 233-6 (2003) 815-820.

[25] J.L. Raphanel, J.H. Schmitt, B. Baudelet, Int. J. Plasticity 2 (1986) 371-378.

[26] E. El-Danaf, S.R. Kalidindi, R.D. Dohert, Metall. Mater. Trans. A 30A (1998) 1223-1233.

[27] S. Asgari, E. El-Danaf, S.R. Kalidindi, R.D. Doherty, Metall. Mater. Trans. A 28 (1997) 1781-1795.

[28] X. Feaugas, Acta Mater. 47 (1999) 3617-3632.

[29] S.R. Kalidindi, Int. J. Plasticity 17 (2001) 837-860.

[30] R.P. Peng, M. Odén, Y.D. Wang, S. Johansson, Mater. Sci. Eng. A 334 (2002) 215-222.

[31] A.A. Salem, S.R. Kalidindi, R.D. Doherty, Acta Mater. 51 (2003) 4225-4237.

[32] S.R. Kalidindi, A.A. Salem, R.D. Doherty, Adv. Eng. Mater. 5 (2003) 229-232.

[33] Z.S. Basinski, M.S. Szczerba, M. Niewczas, J.D. Embury, S.J. Basinski, Rev. Metall. 94 (1997) 1037-1043.

[34] A.B. Douset, R.H. Wagoner, Metall. Trans. 20A (1989) 1483-1493.

[35] A.K. Ghosh, W.A. Backofen, Metall. Trans. 4 (1973) 1113-1123.

[36] M.F. Vieira, J.H. Schmitt, J.J. Grácio, J.V. Fernandes, J. Mater. Process. Technol. 24 (1990) 313-322. 